\title{
HOXA1 enhances the cell proliferation, invasion and metastasis of prostate cancer cells
}

\author{
HAITAO WANG ${ }^{1 *}$, GUANZHONG LIU $^{2 *}$, DAN SHEN $^{3}$, HUAMAO YE $^{3}$, \\ JINMING HUANG ${ }^{4}$, LI JIAO ${ }^{3}$ and YINGHAO SUN ${ }^{3}$ \\ ${ }^{1}$ Department of E.N.T., Second Hospital of Jilin University, Changchun, Jilin 130041; \\ Departments of ${ }^{2}$ Radiology Imaging and ${ }^{3}$ Urology, Changhai Hospital, Second Military Medical University, \\ Shanghai 200433; ${ }^{4}$ Department of Urologic Surgery, The 85th Hospital of PLA, Shanghai 200052, P.R. China
}

Received April 6, 2015; Accepted May 18, 2015

DOI: $10.3892 / o r .2015 .4085$

\begin{abstract}
HOXA1, a member of the HOX gene family, has been implicated in tumor progression. However, the role of HOXA1 in prostate cancer is not well-established. In the present study, we found that HOXA1 was highly expressed in prostate cancer cells. We then repressed the expression of HOXA1 by short hairpin RNA (shRNA) to investigate the function of HOXA1 in prostate cancer cells. Our in vitro data showed that knockdown of HOXA1 attenuated the growth, invasion and migration of prostate cancer DU-145 and PC-3 cells. Furthermore, knockdown of HOXA1 resulted in an increased E-cadherin level and decreased Snail and MMP-3 levels in the DU-145 cells. In addition, knockdown of HOXA1 inhibited activation of ERK1/2 and AKT in the DU-145 cells. Our in vivo data revealed that knockdown of HOXA1 suppressed the growth and metastasis of prostate cancer cells. Collectively, our findings suggest that HOXA1 is involved in the regulation of prostate cancer progression, including cell growth, migration, invasion and metastasis. Thus, downregulation of HOXA1 may be a novel approach for the treatment of prostate cancer.
\end{abstract}

\section{Introduction}

Prostate cancer is one of the most common cancers in men (1). Conventional therapy, including surgery, radiotherapy and chemotherapy may be useful for early stage prostate cancer. However, once the cancer has metastasized to distant organs,

Correspondence to: Dr Yinghao Sun or Dr Li Jiao, Department of Urology, Changhai Hospital, Second Military Medical University, 168 Chang Hai Road, Shanghai 200433, P.R. China

E-mail: yinghksun@163.com

E-mail: 36705487@qq.com

*Contributed equally

Key words: HOXA1, growth, invasion, migration, metastasis, prostate cancer conventional therapy may achieve small improvements in the overall survival of patients with prostate cancer (2). Therefore, a better understanding of the molecular mechanisms involved in prostate cancer progression is essential for the diagnosis and treatment of prostate cancer.

There are 38 HOX genes that have been identified in human cells, and can be divided into 4 groups (HOXA-HOXD). The HOXA gene family has been reported to play a pivotal role in the regulation of several important cellular processes. Overexpression of the HOXA gene family has been observed in several malignant tissues, and is closely associated with the progression of cancer (3-5). Thus, the HOXA gene family may serve as potential targets for antitumor therapy (6). As one important member of HOXA, HOXA1 has been found to be highly expressed in many types of cancer including breast and ovarian cancer, and overexpression of HOXA1 is correlated with tumor progression and poor prognosis $(7,8)$. However, little is known regarding to the biological function of HOXA1 in prostate cancer.

In the present study, we detected the expression of HOXA1 in different prostate cancer cell lines by western blot analysis. We further stably silenced HOXA1 expression in prostate cancer DU-145 and PC-3 cells by short hairpin RNA (shRNA) to evaluate the effect of HOXA1 knockdown on the growth, invasion and metastasis of prostate cancer cells in vitro and in vivo.

\section{Materials and methods}

Antibodies. Mouse monoclonal anti- HOXA1 and anti- $\beta$-actin antibodies were obtained from Santa Cruz Biotechnology (Santa Cruz, CA, USA). Rabbit polyclonal anti-E-cadherin, p-ERK1/2, ERK1/2, p-AKT and AKT, and mouse monoclonal anti-Snail antibodies were purchased from Cell Signaling Technology (Danvers, MA, USA). Mouse monoclonal anti-MMP-3 antibody was purchased from Abcam (Cambridge, MA, USA).

Cell lines and culture conditions. Human prostate cancer cell lines LNCap, PC-3, and DU-145 and normal prostate cell line RPWE-1 were all obtained from the American Type Culture Collection (ATCC; Manassas, VA, USA). Prostate cancer cell 
lines were maintained in RPMI-1640 medium containing $10 \%$ fetal bovine serum (FBS) in a $5 \% \mathrm{CO}_{2}$ atmosphere at $37^{\circ} \mathrm{C}$. RPWE-1 cells were maintained in K-SFM medium containing $10 \% \mathrm{FBS}$ in a $5 \% \mathrm{CO}_{2}$ atmosphere at $37^{\circ} \mathrm{C}$.

Western blot analysis. Cells were washed with cold PBS and then lysed in RIPA lysis buffer containing protease inhibitor. Total protein concentration was determined using the BCA protein assay kit (Pierce Company, Rockford, IL, USA). Equal amounts of total protein $(\sim 50 \mu \mathrm{g})$ were separated by SDS-polyacrylamide separating gels and then transferred onto PVDF membranes. Then the PVDF membranes were blocked with TBS-T buffer containing 5\% BSA. The PVDF membranes were probed with the primary antibodies against HOXA1 (1:500), E-cadherin (1:1,000), Snail (1:1,000), MMP-3 (1:500), p-ERK1/2 (1:1,000), ERK1/2 (1:1,000), p-AKT(1:1,000), AKT $(1: 1,000)$ or $\beta$-actin $(1: 1,000)$ and incubated at $4^{\circ} \mathrm{C}$ overnight. Next, the membranes were washed in TBS-T for 3 times and then incubated with either the anti-mouse or anti-rabbit horseradish peroxidase-conjugated secondary antibody for $1 \mathrm{~h}$ at room temperature. The membranes were further treated with enhanced chemiluminescence (Pierce Company) and the bands were exposed to X-ray film. Densitometric analysis of the bands was performed with Quantity One software (Bio-Rad, Hercules, CA, USA).

Knockdown of HOXAl expression by shRNA. shRNAs were designed and obtained from Genchem Biotechnology Company (Shanghai, China). Briefly, 4 different shRNAs targeting the HOXA1 gene (ShRNA\#1, ShRNA\#2, ShRNA\#3 and ShRNA\#4) were ligated into the pGPU6/Neo vector for expressing HOXA1 shRNA. A scrambled sequence was ligated into the pGPU6/Neo vector and used as the negative control (NC) shRNA. DU-145 and PC-3 cells were transfected with NC, ShRNA\#1, ShRNA\#2, ShRNA\#3 or ShRNA\#4, respectively. G418 selection was used to isolate the stable cell clones (Gibco, Grand Island, NY, USA).

Cell count assay. Cells were seeded into a 24-well plate at a density of $1 \times 10^{4}$ cells/well and then incubated in medium for the indicated times. Then the medium was removed and cells were trypsinized. After staining with trypan blue, the cell number was counted in a hemocytometer. Each sample was measured in triplicate and repeated 3 times.

MTT assay. Cells were seeded in a 96-well plate in triplicate at a concentration of $1 \times 10^{3}$ cells/well. On the indicated day, the medium was removed and MTT solution was added to the wells. Four hours later, DMSO was added and the cells were further incubated for $10 \mathrm{~min}$ with gentle shaking at $37^{\circ} \mathrm{C}$. Finally, the optical density of each well was measured using a microplate reader (Bio-Rad) at $490 \mathrm{~nm}$.

Invasion and migration assays. A 24-well Transwell plate with $8.0-\mu \mathrm{m}$ pore size polycarbonate membranes was purchased from Costar (Corning Incorporated, Corning, NY, USA) and used in the invasion and migration assays. In the in vitro invasion assay, the upper chambers were coated with $50 \mu 1$ Matrigel diluted with serum-free medium. Cells were resuspended in RPMI-1640 medium containing 0.5\% FBS at a density of $5 \times 10^{5}$ cells $/ \mathrm{ml}$. Then $200 \mu \mathrm{l}$ of cell suspension was loaded into the upper chambers and the lower chambers were filled with $500 \mu 1$ of RPMI-1640 medium containing $20 \%$ FBS. After $24 \mathrm{~h}$ of incubation, the cells that invaded through the Matrigel-coated filters were fixed and stained with crystal violet. The number of invaded cells was counted under a microscope at $\mathrm{x} 50$ magnification. In the in vitro migration assay, the upper chambers were not coated with Matrigel. Cells $\left(1 \times 10^{5}\right.$ cells in $\left.200 \mu 1\right)$ were seeded into the upper chambers and $500 \mu \mathrm{l}$ of RPMI-1640 medium containing 20\% FBS was added into the lower chambers. Twenty-four hours later, the migrated cells were stained with crystal violet and counted by light microscope at $\mathrm{x} 50$ magnification. Three independent experiments were performed for the invasion and migration assays.

In vivo growth and metastasis assay. Male $\mathrm{BABL} / \mathrm{c}$ nude mice (6-weeks old) were maintained in pathogen-free conditions. All procedures were conducted following the Animal Care and Use Committee guidelines of the Second Military Medical University. The mice were randomly separated into 3 groups $(n=8)$. DU-145 cells at a density of $2.0 \times 10^{5}$ cells $/ 100 \mu 1$ were subcutaneously injected in the back of mice. The length (L) and width (W) of the tumors in the mice were measured with calipers on week 1, 3, 5 and 7. The tumor volume (V) was calculated with the formula: $\mathrm{V}=\left(\right.$ length $\mathrm{x}$ width $\left.{ }^{2}\right) / 2$. Eight weeks later, the mice were sacrificed and tumor weights were measured. Next, the lungs and livers were fixed in $4 \%$ paraformaldehyde and then were sectioned into slices. Each slice was further stained with haematoxylin and eosin (H\&E) and observed under a light microscope to examine the micrometastasis in the lungs and livers.

Statistical analysis. All experimental data are expressed as mean \pm SD. Statistical significance was determined by Student's t-test between two groups or one-way analysis of variance (ANOVA) among multiple groups. Fisher's exact test was performed to compare proportions between groups. $\mathrm{P}<0.05$ was considered to indicate a statistically significant result.

\section{Results}

Expression of HOXAl in prostate cancer cells and knockdown of HOXAl expression by shRNA. We first examined the expression of HOXA1 in the prostate cancer cells. Using western blot analysis, we found that compared with normal prostate RPWE-1 cells, the protein level of HOXA1 was highly expressed in the LNCap, PC-3 and DU-145 cells (Fig. 1A), indicating that HOXA1 may be involved in the progression of prostate cancer. In addition, the highest expression of HOXA1 was observed in the DU-145 cells. To investigate the role of HOXA1 in prostate cancer cells, we transfected DU-145 and PC-3 cells with either a control shRNA vector (NC) or 4 different HOXA1 shRNAs (ShRNA\#1, ShRNA\#2, ShRNA\#3 and ShRNA\#4), respectively. Stable cell clones were isolated, and the knockdown efficiency was tested by western blotting. We found that all HOXA1 shRNAs suppressed the expression of HOXA1 in the DU-145 and PC-3 cells. Relative expression of HOXA1 relative to the Mock cells was: for DU-145: 
A

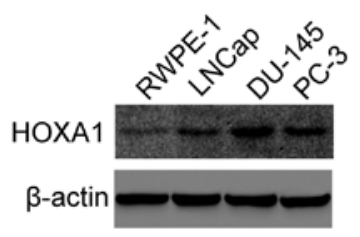

B

B

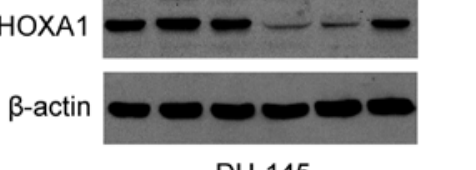

DU-145

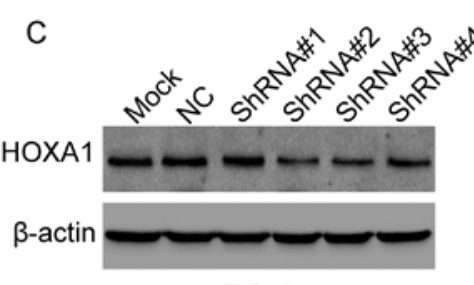

PC-3
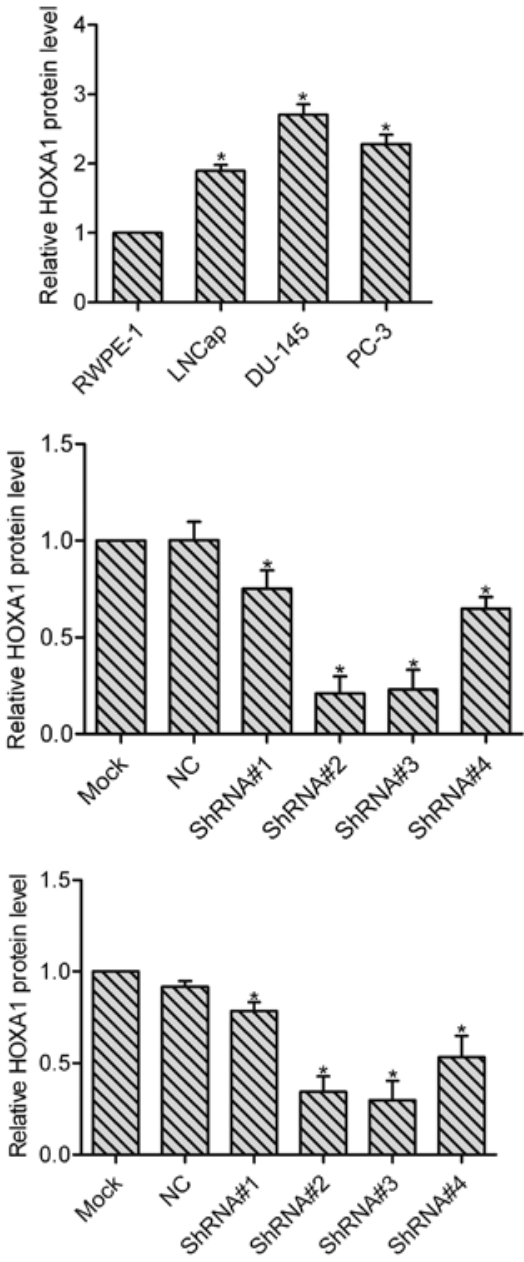

Figure 1. HOXA1 expression in prostate cancer cells is silenced by shRNA. (A) The protein level of HOXA1 in human normal prostate RPWE-1 cells and prostate cancer LNCap, PC-3 and DU-145 cells was detected by western blot analysis. (B) DU-145 cells and (C) PC-3 cells were transfected with mock, negative control (NC) or 4 different HOXA1 shRNAs (ShRNA\#1, ShRNA\#2, ShRNA\#3 and ShRNA\#4), respectively. Knockdown efficiency was determined by western blot analysis. Data are expressed as mean \pm SD of 3 experiments ("p $<0.05)$. shRNA, short hairpin RNA.

A

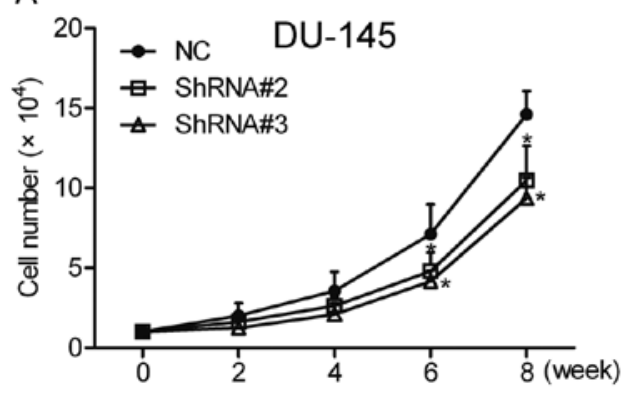

C

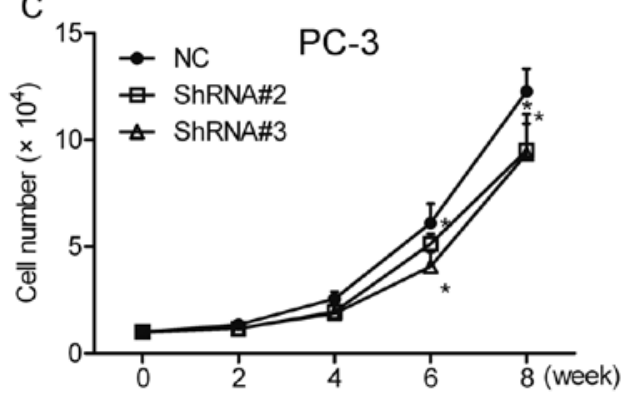

B

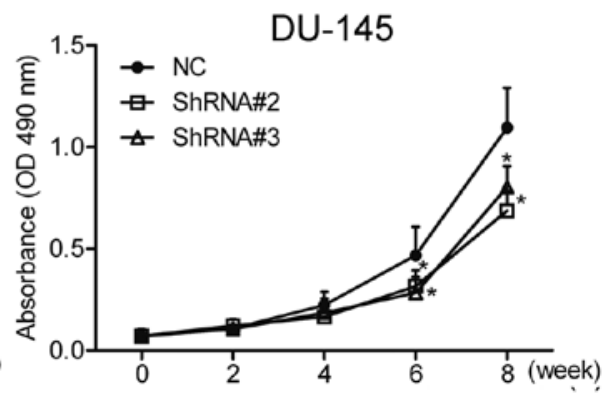

D

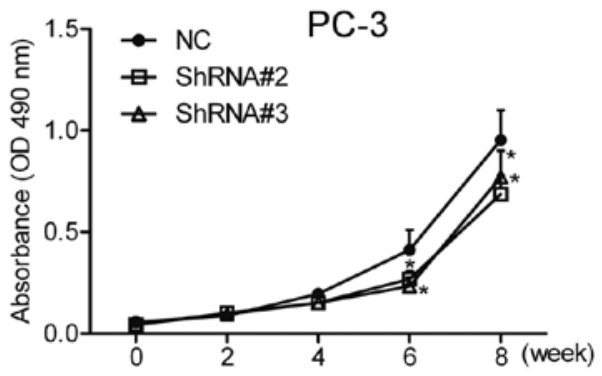

Figure 2. Effects of HOXA1 knockdown on the growth of prostate cancer cells in vitro. (A and C) Cell count assays were performed to analyze the effect of HOXA1 knockdown on prostate cancer cell growth in vitro. (B and D) MTT assays were performed to analyze the effect of HOXA1 knockdown on prostate cancer cell growth in vitro. Data are expressed as mean $\pm \mathrm{SD}$ of 3 experiments $(* \mathrm{p}<0.05)$. 
A
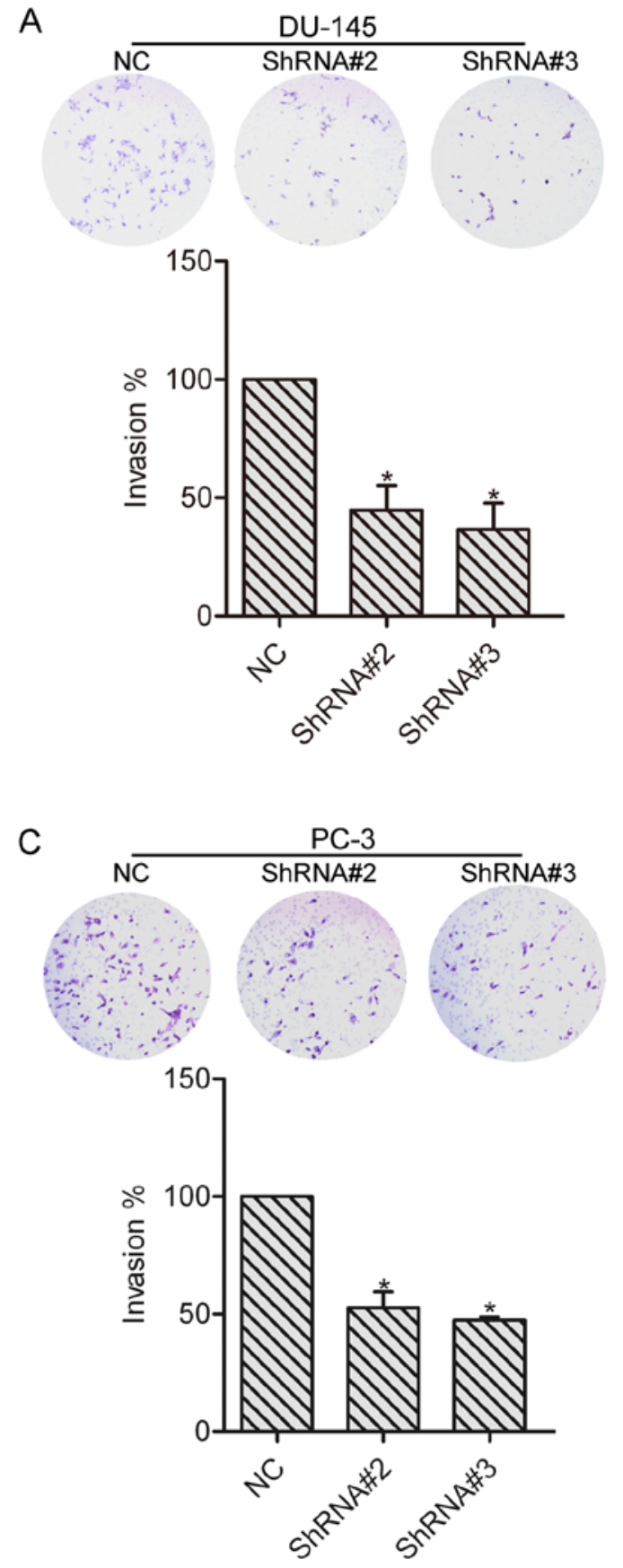

B
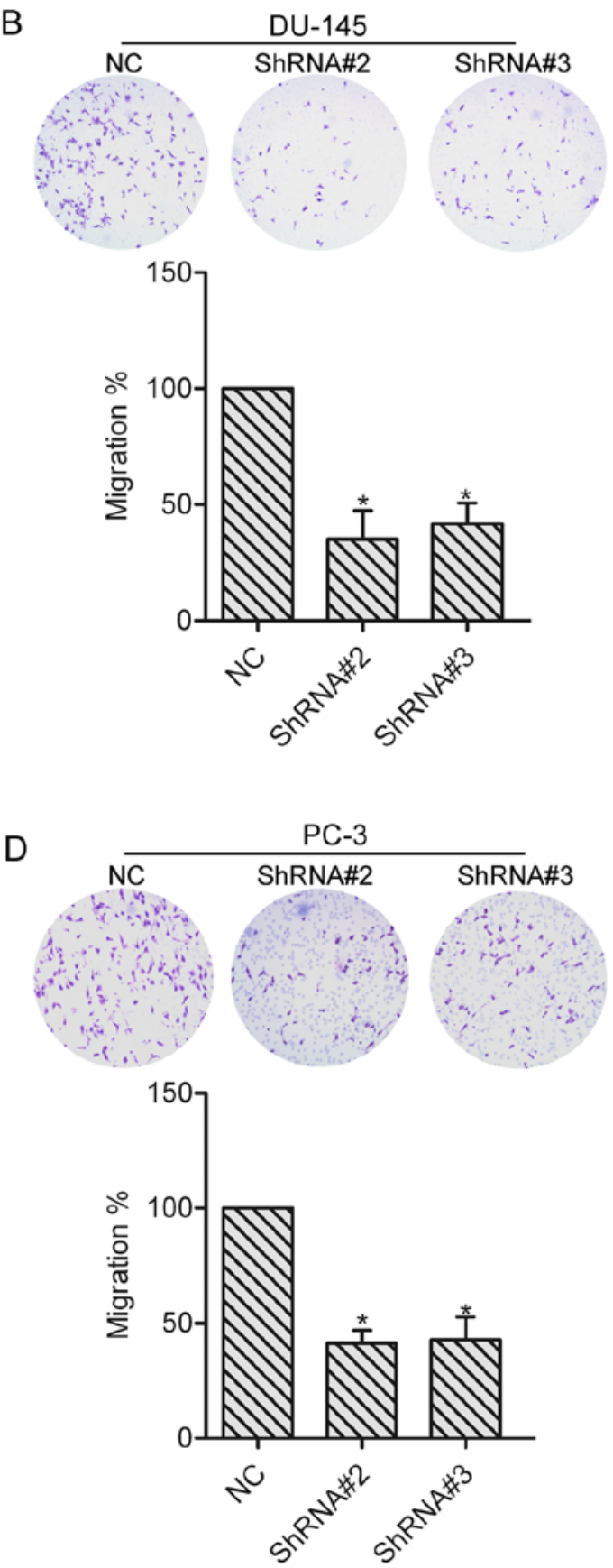

Figure 3. Effects of HOXA1 knockdown on the invasion and migration of prostate cancer cells in vitro. Cells were allowed to invade or migrate for $24 \mathrm{~h}$, and then the invaded or migrated cells were fixed, stained and counted. (A) Knockdown of HOXA1 suppressed the invasion of DU-145 cells in vitro. (B) Knockdown of HOXA1 suppressed the migration of DU-145 cells in vitro. (C) Knockdown of HOXA1 suppressed the invasion of PC-3 cells in vitro. (D) Knockdown of HOXA1 suppressed the migration of PC-3 cells in vitro. Data are expressed as mean \pm SD of 3 experiments ("p $<0.05$ ).

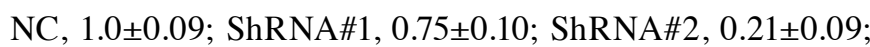
ShRNA\#3, 0.23 \pm 0.10 and ShRNA\#4, 0.65 \pm 0.06 ; for PC-3: NC, 0.93 \pm 0.02 ; ShRNA\#1, 0.78 \pm 0.03 ; ShRNA\#2, 0.31 \pm 0.05 ; ShRNA\#3, $0.30 \pm 0.06$ and ShRNA\#4, $0.57 \pm 0.07$ (Fig. 1B and C). As RNAi efficiency was much higher in the ShRNA\#2 and ShRNA\#3 transfected cells, we then used ShRNA\#2 and ShRNA\#3 transfected cells in the subsequent experiments.

Role of HOXAl in prostate cancer cell growth in vitro. Cell count and MTT assays were performed to investigate the effect of HOXA1 knockdown on prostate cancer cell growth.
The results showed that compared with the NC cells, knockdown of HOXA1 by shRNA greatly inhibited the growth of the DU-145 and PC-3 cells (Fig. 2). These data suggest that HOXA1 is involved in the growth of prostate cancer cells.

HOXA1 participates in the invasion and migration of prostate cancer cells in vitro. To explore the role of HOXA1 in prostate cancer cell invasion and migration, invasion and migration assays were performed in the NC, ShRNA\#2 and ShRNA\#3 transfected cells. DU-145 and PC-3 cells were allowed to invade or migrate for $24 \mathrm{~h}$, and images were captured to count 

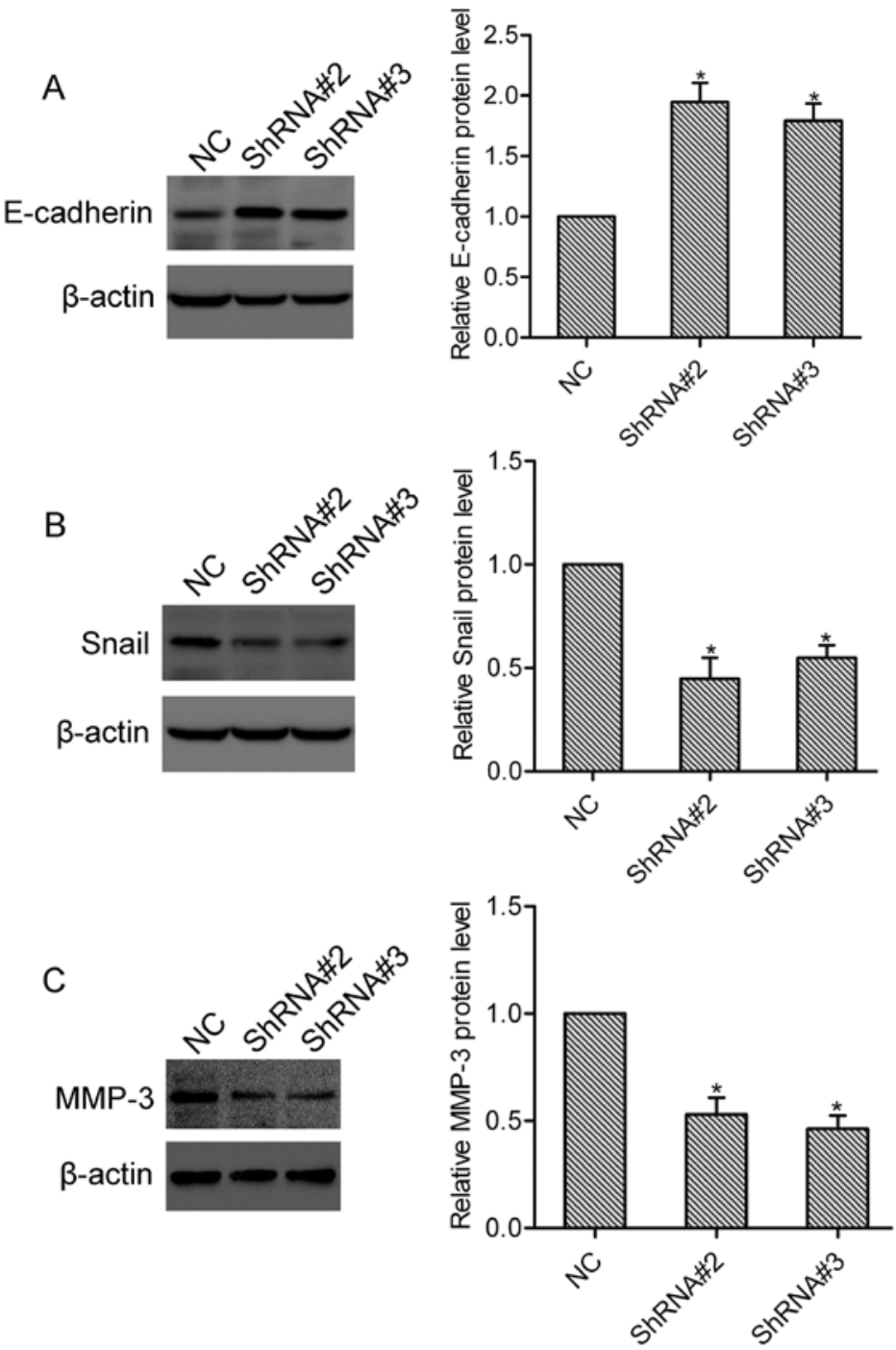

Figure 4. Effects of HOXA1 knockdown on the protein levels of E-cadherin, Snail and MMP-3 in DU-145 cells. Western blot analysis was performed to evaluate whether HOXA1 knockdown affects the protein levels of (A) E-cadherin, (B) Snail and (C) MMP-3. Data are expressed as mean \pm SD of 3 experiments $\left({ }^{*} \mathrm{p}<0.05\right)$.

the number of cells. The results showed that knockdown of HOXA1 by shRNA markedly reduced the invasion and migration abilities as compared to the NC cells (Fig. 3), suggesting the involvement of HOXA1 in prostate cancer cell invasion and migration. The following experiments were performed with DU-145 cells.

HOXA1 regulates the expression of E-cadherin, Snail and $M M P-3$. E-cadherin and Snail are well-established epithelialmesenchymal transition (EMT) markers in cancer, and participate in the regulation of tumor invasion and metastasis (9). To investigate the effect of HOXA1 on E-cadherin and Snail expression, DU-145 cells transfected with or without HOXA1 shRNA were collected and subjected to western blotting. Here, western blot analysis showed that compared with the NC cells, the expression of E-cadherin was upregulated, while the expression of Snail was downregulated in both the ShRNA\#2 and ShRNA\#3 transfected cells (Fig. 4A and B). These data suggest that HOXA1 regulates the expression of E-cadherin and Snail in prostate cancer cells. MMP-3 is a crucial modulator in tumor invasion and metastasis (10). Using western blotting, we found that knockdown of HOXA1 significantly suppressed the expression of MMP-3 in the DU-145 cells (Fig. 4C), indicating that HOXA1 affects the expression of MMP-3 in prostate cancer cells.

HOXA1 induces activation of ERK1/2 and AKT. We further detected the function of HOXA1 in the activation of ERK1/2 and AKT, which both play vital roles in the progression of prostate cancer. Notably, western blot analysis showed that knockdown of HOXA1 in the DU-145 cells markedly inhibited activation of ERK1/2 and AKT (Fig. 5A and B).

Knockdown of HOXAl suppresses prostate cancer cell growth and metastasis in vivo. To determine the effect of HOXA1 knockdown on cell growth and metastasis in vivo, an in vivo tumor xenograft model was used, and DU-145 cells transfected with or without HOXA1 shRNA were injected subcutaneously into the back of the mice, respectively. An in vivo growth curve showed that the tumor size in the 

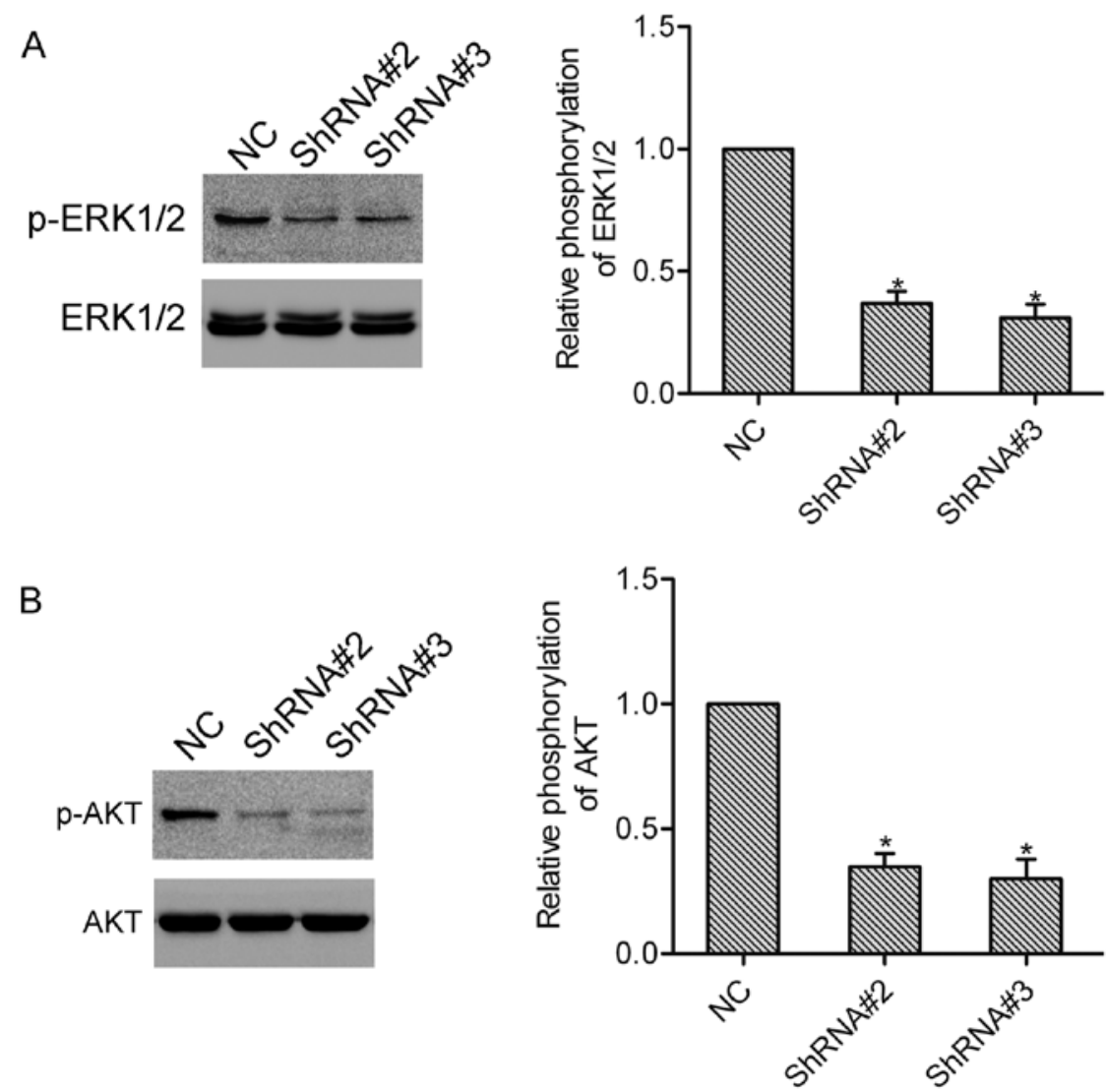

Figure 5. Effects of HOXA1 knockdown on activation of ERK1/2 and AKT in DU-145 cells. Western blot analysis was performed to evaluate whether HOXA1 knockdown affects the phosphorylation of (A) ERK1/2 and (B) AKT. Data are expressed as mean \pm SD of 3 experiments ("p<0.05).

ShRNA\#2 or ShRNA\#3 group was much smaller than that in the NC group (Fig. 6A). Eight weeks later, the mice were sacrificed and the mean tumor weight was calculated. The mean tumor weights in the NC, ShRNA\#2 and ShRNA\#3 groups were $4.59 \pm 1.82,2.28 \pm 0.37$ and $1.86 \pm 0.21 \mathrm{~g}$, respectively (Fig. 6B). In agreement with the tumor volume results, the mean tumor weights in the ShRNA\#2 and ShRNA\#3 groups were decreased as compared to that in the NC group $(\mathrm{P}<0.05)$. These data suggest that knockdown of HOXA1 suppressed the growth of prostate cancer cells. Furthermore, we found that liver metastasis was 75\% (6/8) in mice injected with the NC cells. However, liver metastasis in mice injected with the ShRNA\#2 and ShRNA\#3 cells was 25\% (2/8) and $37.5 \%(3 / 8)$, respectively. In addition, micrometastasis in the liver section was counted under a microscope, and the results showed that the number of micrometastases was greatly decreased in the ShRNA\#2 and ShRNA\#3 groups as compared to the NC group (Fig. 6C). However, lung metastasis was not observed in either the NC group or the ShRNA\#2 and ShRNA\#3 groups. Together, these findings support the notion that HOXA1 participates in the growth and metastasis of prostate cancer cells in vivo.

\section{Discussion}

Overexpression of HOXA1 has been observed in breast cancer and oral squamous cell cancer, and a high HOXA1 expression level correlates with poor prognosis in patients with cancer $(8,11)$. In the present study, we found that the protein level of HOXA1 was highly expressed in prostate cancer cells, suggesting a possible role of HOXA1 in prostate cancer progression.

It has been reported that HOXA1 can alter cell proliferation and the cell cycle in non-small cell lung cancer cells $(12,13)$. However, a recent study demonstrated that enforced expression of HOXA1 in lung H69AR cells increased cell apoptosis and cell cycle arrest (14). The effect of HOXA1 on prostate cancer cell growth is largely unknown. Here, we found that knockdown of HOXA1 suppressed the growth of prostate cancer DU-145 and PC-3 cells in vitro. Moreover, Brock et al demonstrated that knockdown of HOXA1 reduced tumor growth in a mouse xenograft model of mammary tumor progression (15). Here, we found that knockdown of HOXA1 suppressed the tumor growth of human prostate cancer DU-145 cells in vivo. These data suggest that HOXA1 contributes to cell proliferation in prostate cancer cells. It has been reported that HOXA1 promotes the invasion and migration of hepatocellular carcinoma cells (7), and is required for the metastasis and tumorigenesis of melanoma cancer cells (16). However, the function of HOXA1 in prostate cancer cell invasion and metastasis remains unclear. Here, for the first time, our results showed that knockdown of HOXA1 in prostate cancer cells suppressed the invasion and migration in vitro, and inhibited the metastasis in vivo, supporting the conclusion that HOXA1 is involved in the regulation of invasion and metastasis in prostate cancer cells. 



Figure 6. Effects of HOXA1 knockdown on prostate cancer cell growth and metastasis in vivo. Negative control (NC), HOXA1 shRNA\#2 (ShRNA\#2) and HOXA1 shRNA\#3 (ShRNA\#3) cells were injected subcutaneously into 3 groups of nude mice $(n=8)$. (A) Tumor volume was measured at week 1, 3, 5 and 7 . (B) Eight weeks later, the mice were sacrificed. The weight of the tumor in each group was measured and the mean tumor weight was calculated. (C) Following staining with H\&E, micrometastasis in the liver was observed and counted under a microscope ( $\left.{ }^{*} \mathrm{p}<0.05\right)$ (arrow, micrometastasis). shRNA, short hairpin RNA; $\mathrm{H} \& \mathrm{E}$, haematoxylin and eosin.

Epithelial-mesenchymal transition (EMT) is regarded as a critical step in tumor metastasis, and E-acdherin and Snail are both EMT markers in cancer. It is well known that E-cadherin, which can be transcriptionally repressed by Snail, mediates cell-cell adhesion and is essential for the invasion and metastasis of prostate cancer (17). Studies have found that knockdown of HOXD3, a member of the HOX gene family, increased the expression of E-cadherin in lung cancer cells $(18,19)$. Zhang et al showed that HOXA1 was required for the E-cadherin-dependent anchorage-independent survival of human mammary carcinoma cells (20). However, whether HOXA1 knockdown can influence the expression level of E-cadherin and Snail in prostate cancer cells is still unclear. Our study showed that knockdown of HOXA1 upregulated the protein level of E-cadherin, and downregulated the protein level of Snail in the DU-145 cells, suggesting that HOXA1 may regulate EMT in prostate cancer cells. Belonging to the matrix metalloproteinase (MMP) family, MMP-3 can degrade various components of the extracellular matrix (ECM), and plays a crucial role in tumor invasion and metastasis (21). Using shRNA technology, we found that HOXA1 regulated the expression of MMP-3 in prostate cancer cells. As we proved that downregulation of HOXA1 can suppress prostate cancer cell invasion and metastasis, these data indicate that HOXA1 may promote the invasion and metastasis of prostate cancer cells via the regulation of E-cadherin, Snail and MMP-3 expression.

It is well known that the ERK1/2 and AKT signaling pathways both are pivotal players in the progression of cancer $(22,23)$. Many studies have confirmed that activation of ERK1/2 as well as AKT regulates tumor cell processes, including growth, migration, apotosis, invasion and metas- 
tasis $(24,25)$. Hou et al showed that increased expression of HOXA1 enhanced the phosphorylation of ERK1/2 in breast cancer MCF-7 cells (26). In the present study, we found that knockdown of HOXA1 inhibited activation of ERK1/2 and AKT in DU-145 cells. These data indicate that HOXA1 may affect the progression of prostate cancer cells via the ERK $1 / 2$ and AKT pathways.

In summary, our findings showed that HOXA1 was highly expressed in prostate cancer cells. Knockdown of HOXA1 by shRNA inhibited the growth, invasion and migration of prostate cancer cells. Furthermore, knockdown of HOXA1 affected the expression level of E-cadherin, Snail and MMP-3, and induced the activation of ERK $1 / 2$ and AKT in vitro. In addition, knockdown of HOXA1 suppressed the growth and metastasis of prostate cancer cells in vivo. Together, the present study indicates that downregulation of HOXA1 can inhibit the progression of prostate cancer cells. Thus, HOXA1 may be a promising target for the treatment of prostate cancer.

\section{Acknowledgements}

This study was supported by the National Natural Science Foundation of China (nos. 81202036 and 81272818) and the Postdoctoral Funds of China (2013M532143 and 2013M542435).

\section{References}

1. Siegel R, Ma J, Zou Z and Jemal A: Cancer statistics, 2014. CA Cancer J Clin 64: 9-29, 2014.

2. Chung LW, Baseman A, Assikis V and Zhau HE: Molecular insights into prostate cancer progression: The missing link of tumor microenvironment. J Urol 173: 10-20, 2005.

3. Zhang ML, Nie FQ, Sun M, Xia R, Xie M, Lu KH and Li W: HOXA5 indicates poor prognosis and suppresses cell proliferation by regulating p21 expression in non small cell lung cancer. Tumour Biol: Dec 31, 2014 (Epub ahead of print).

4. Ma RL, Shen LY and Chen KN: Coexpression of ANXA2, SOD2 and HOXA13 predicts poor prognosis of esophageal squamous cell carcinoma. Oncol Rep 31: 2157-2164, 2014.

5. Yamatoji M, Kasamatsu A, Yamano Y, Sakuma K, Ogoshi K, Iyoda M, Shinozuka K, Ogawara K, Takiguchi Y, Shiiba M, et al: State of homeobox A10 expression as a putative prognostic marker for oral squamous cell carcinoma. Oncol Rep 23: 61-67, 2010.

6. Liu XH, Lu KH, Wang KM, Sun M, Zhang EB, Yang JS, Yin DD, Liu ZL, Zhou J, Liu ZJ, et al: MicroRNA-196a promotes non-small cell lung cancer cell proliferation and invasion through targeting HOXA5. BMC Cancer 12: 348, 2012.

7. Zha TZ, Hu BS, Yu HF, Tan YF, Zhang Y and Zhang K: Overexpression of HOXA1 correlates with poor prognosis in patients with hepatocellular carcinoma. Tumour Biol 33 2125-2134, 2012.

8. Bitu CC, Destro MF, Carrera M, da Silva SD, Graner E, Kowalski LP, Soares FA and Coletta RD: HOXA1 is overexpressed in oral squamous cell carcinomas and its expression is correlated with poor prognosis. BMC Cancer 12: 146, 2012.
9. Al Saleh S, Sharaf LH and Luqmani YA: Signalling pathways involved in endocrine resistance in breast cancer and associations with epithelial to mesenchymal transition (Review). Int J Oncol 38: 1197-1217, 2011.

10. McDonnell S and Matrisian LM: Stromelysin in tumor progression and metastasis. Cancer Metastasis Rev 9: 305-319, 1990.

11. Chariot A and Castronovo V: Detection of HOXA1 expression in human breast cancer. Biochem Biophys Res Commun 222: 292-297, 1996

12. Cho HS, Toyokawa G, Daigo Y, Hayami S, Masuda K, Ikawa N, Yamane Y, Maejima K, Tsunoda T, Field HI, et al: The JmjC domain-containing histone demethylase KDM3A is a positive regulator of the G1/S transition in cancer cells via transcriptional regulation of the HOXA1 gene. Int J Cancer 131: E179-E189, 2012.

13. Zhan M, Qu Q, Wang G, Liu YZ, Tan SL, Lou XY, Yu J and Zhou HH: Let-7c inhibits NSCLC cell proliferation by targeting HOXA1. Asian Pac J Cancer Prev 14: 387-392, 2013.

14. Xiao F, Bai Y, Chen Z, Li Y, Luo L, Huang J, Yang J, Liao H and Guo L: Downregulation of HOXA1 gene affects small cell lung cancer cell survival and chemoresistance under the regulation of miR-100. Eur J Cancer 50: 1541-1554, 2014.

15. Brock A, Krause S, Li H, Kowalski M, Goldberg MS, Collins JJ and Ingber DE: Silencing HoxA1 by intraductal injection of siRNA lipidoid nanoparticles prevents mammary tumor progression in mice. Sci Transl Med 6: 217ra2, 2014.

16. Wardwell-Ozgo J, Dogruluk T, Gifford A, Zhang Y,Heffernan TP, van Doorn R, Creighton CJ, Chin L and Scott KL: HOXA1 drives melanoma tumor growth and metastasis and elicits an invasion gene expression signature that prognosticates clinical outcome. Oncogene 33: 1017-1026, 2014.

17. Lee MY and Shen MR: Epithelial-mesenchymal transition in cervical carcinoma. Am J Transl Res 4: 1-13, 2012.

18. Ohta H, Hamada J, Tada M, Aoyama T, Furuuchi K, Takahashi Y, Totsuka Y and Moriuchi T: HOXD3-overexpression increases integrin alpha v beta 3 expression and deprives E-cadherin while it enhances cell motility in A549 cells. Clin Exp Metastasis 23: 381-390, 2006.

19. Hamada Ji, Omatsu T, Okada F, Furuuchi K, Okubo Y, Takahashi Y, Tada M, Miyazaki YJ, Taniguchi Y, Shirato H, et al: Overexpression of homeobox gene HOXD3 induces coordinate expression of metastasis-related genes in human lung cancer cells. Int J Cancer 93: 516-25, 2001.

20. Zhang X, Emerald BS, Mukhina S, Mohankumar KM, Kraemer A, Yap AS, Gluckman PD, Lee KO and Lobie PE: HOXA1 is required for E-cadherin-dependent anchorageindependent survival of human mammary carcinoma cells. J Biol Chem 281: 6471-6481, 2006.

21. Johansson N, Ahonen M and Kähäri VM: Matrix metalloproteinases in tumor invasion. Cell Mol Life Sci 57: 5-15, 2000.

22. Santarpia L, Lippman SM and El-Naggar AK: Targeting the MAPK-RAS-RAF signaling pathway in cancer therapy. Expert Opin Ther Targets 16: 103-119, 2012.

23. Edlind MP and Hsieh AC: PI3K-AKT-mTOR signaling in prostate cancer progression and androgen deprivation therapy resistance. Asian J Androl 16: 378-386, 2014.

24. Torii S, Yamamoto T, Tsuchiya Y and Nishida E: ERK MAP kinase in $\mathrm{G}$ cell cycle progression and cancer. Cancer Sci 97: 697-702, 2006.

25. Kumar A, Rajendran V, Sethumadhavan R and Purohit R: AKT kinase pathway: A leading target in cancer research. Sci World J 2013: 756134, 2013.

26. Hou L, Xu B, Mohankumar KM, Goffin V, Perry JK, Lobie PE and Liu DX: The prolactin receptor mediates HOXA1-stimulated oncogenicity in mammary carcinoma cells. Int J Oncol 41: 2285-2295, 2012 